\title{
Survival trends for extranodal NK/T-cell lymphoma, nasal type from different anatomical sites: a population-based study
}

\author{
Lu He $^{1,2 \#}$, Yixin Zou ${ }^{1 \#}$, Xiaolu Tang ${ }^{1}$, Jia Wang ${ }^{3}$, Lingxiao Xing ${ }^{1}$, Jing Zhang ${ }^{1}$, Jianyong Li $^{1,4}$, Jingjing Guo ${ }^{5}$, \\ Yi Miao ${ }^{1}$
}

${ }^{1}$ Department of Hematology, the First Affiliated Hospital of Nanjing Medical University, Jiangsu Province Hospital, Nanjing, China; ${ }^{2}$ Department of Pathology, Nanjing Drum Tower Hospital, The Affiliated Hospital of Nanjing University Medical School, Nanjing, China; ${ }^{3}$ Department of Nuclear Medicine, the First Affiliated Hospital of Nanjing Medical University, Jiangsu Province Hospital, Nanjing, China; ${ }^{4}$ National Clinical Research Center for Hematologic Diseases, the First Affiliated Hospital of Soochow University, Suzhou, China; ${ }^{5}$ Department of Oncology, The Second Hospital of Nanjing, Nanjing University of Chinese Medicine, Nanjing, China

Contributions: (I) Conception and design: Y Miao, J Li; (II) Administrative support: J Guo; (III) Provision of study materials or patients: L Xing, J Zhang; (IV) Collection and assembly of data: X Tang, J Wang; (V) Data analysis and interpretation: L He, Y Zou; (VI) Manuscript writing: All authors; (VII) Final approval of manuscript: All authors.

"These authors contributed equally to this work.

Correspondence to: Dr. Yi Miao. Department of Hematology, the First Affiliated Hospital of Nanjing Medical University, Jiangsu Province Hospital, Nanjing 210029, China. Email: miaoyi1992@hotmail.com; Dr. Jingjing Guo. Department of Oncology, The Second Hospital of Nanjing, Nanjing University of Chinese Medicine, 1-1 Zhongfu Road, Nanjing 210003, China. Email: guojingjing0017@126.com; Dr. Jianyong Li. Department of Hematology, the First Affiliated Hospital of Nanjing Medical University, Jiangsu Province Hospital, Nanjing 210029, China. Email: lijianyonglm@126.com.

Background: Extranodal natural killer/T-cell lymphoma (NKTCL), nasal type mostly involves the upper aerodigestive tract (UAT). NKTCLs derived from the UAT are referred to as nasal NKTCLs, while those without UAT involvement are referred to as extra-nasal NKTCLs. In this study, we aimed to investigate the outcomes and survival trends of NKTCLs from different anatomical sites.

Methods: Data from the US Surveillance, Epidemiology, and End Results (SEER) database on NKTCL (diagnosed between 1987 and 2016) were retrospectively analyzed.

Results: A total of 714 patients with NKTCL were included. The median overall survival (OS) and cancerspecific survival (CSS) were 36 and 57 months, respectively. For the entire cohort, the OS was improved from era 1 to era 2 with marginal significance $(\mathrm{P}=0.0595)$, however, no improvement was shown in CSS. For nasal NKTCLs, the OS of patients from era 2 was significantly improved compared to that of patients from era 1 ( $\mathrm{P}=0.0244)$. The OS was significantly improved in non-cavity nasal NKTCLs $(\mathrm{P}=0.031)$ but not in nasal cavity NKTCLs $(\mathrm{P}=0.2982)$. Significant improvements in OS $(\mathrm{P}=0.0025)$ and $C S S(\mathrm{P}=0.0176)$ were found in stage I/II non-cavity nasal NKTCLs. For patients with extra-nasal NKTCLs, no difference was found in survival outcomes between the 2 eras.

Conclusions: We have demonstrated that the outcomes of non-cavity nasal NKTCLs, especially those in stage I/II, have improved in the new era, while the outcomes of nasal cavity NKTCLs and extra-nasal NKTCLs have not improved. Our study highlights the heterogeneity in clinical outcomes and biology among NKTCLs from different sites. More studies are warranted to define the optimal treatments for patients with NKTCLs.

Keywords: Survival trends; natural killer/T-cell lymphoma (NKTCL); nasal; extra-nasal; Surveillance, Epidemiology, and End Results database (SEER database)

^ ORCID: 0000-0003-2749-7857. 
Submitted Mar 08, 2021. Accepted for publication Apr 29, 2021.

doi: $10.21037 /$ atm-21-1748

View this article at: http://dx.doi.org/10.21037/atm-21-1748

\section{Introduction}

Extranodal natural killer/T-cell lymphoma (NKTCL), nasal type is a predominantly extranodal lymphoma with a highly aggressive clinical course. It always has an extranodal presentation. The upper aerodigestive tract (UAT) is most commonly involved, with the nasal cavity being the typical site of involvement. NKTCL from the UAT is referred to as nasal NKTCL, while NKTCL occurring outside the UAT is usually referred to as extra-nasal (or non-nasal) NKTCL, preferential sites of which include the skin, soft tissue, the gastrointestinal (GI) tract, and the testes $(1,2)$. Nasal NKTCLs frequently present as stage I/II disease with favorable outcomes, while extra-nasal NKTCLs often present as stage III/IV disease with more aggressive clinical features and inferior survival $(1,3,4)$.

Historically, the prognosis of NKTCLs, especially those in the advanced stage (stage III/IV), has been poor. The emergence and application of modern therapies (e.g., asparaginase-based regimens) have possibly improved the outcomes of patients with NKTCL since 2010, however, these improved outcomes attributed to modern therapies have not been demonstrated in randomized trials (5-7). The study by Yamaguchi et al. demonstrated that the prognosis of nasal NKTCLs but not extra-nasal NKTCLs improved in the new era, suggesting that the survival benefits provided by modern therapies were different for nasal and extra-nasal NKTCLs (8). Nevertheless, this study was based on an Asian population, and whether the findings from this study can be replicated in Western populations remains unknown. Additionally, cases of nasal NKTCLs could be classified into nasal cavity NKTCLs and NKTCLs from other UAT sites, and a previous study suggested that these 2 subgroups had different clinical characteristics, immunophenotypes, and survival outcomes (9). Whether the survival trends of these two subgroups are similar or different remains to be determined. Therefore, we conducted a retrospective study using the US Surveillance, Epidemiology, and End Results (SEER) database (1987 to 2016) to analyze the trends in survival for nasal and extra-nasal NKTCLs. We also stratified these patients according to different parameters to study the variance in survival trends among different subgroups. We present the following article in accordance with the STROBE reporting checklist (available at http:// dx.doi.org/10.21037/atm-21-1748).

\section{Methods}

\section{SEER database}

The SEER database is representative of the US population, with patient-level data abstracted from 18 geographically diverse populations that represent rural, urban, and regional populations (10). NKTCL cases between 1987 and 2016 in the SEER public access database and their corresponding details were retrieved with the use of SEER ${ }^{*}$ Stat version 8.3.8 software. Patients were uniformly reviewed and staged according to the Ann Arbor staging system. All of the SEER data are de-identified and analysis of the data does not require institutional review board approval or informed consent from the patients. The study was conducted in accordance with the Declaration of Helsinki (as revised in 2013).

\section{Patient selection}

The International Classification of Diseases for Oncology, 3rd Edition (ICD-O-3) code 9719 was used to identify cases of NKTCL. Patients with primary tumor sites documented as lymph nodes were excluded. Other exclusion criteria included cases without histological confirmation, incomplete survival data, recorded survival time of zero (0) months, missing/unknown cause of death (COD), and extranodal NKTCL not as the first primary malignancy (Figure S1).

\section{Statistical analysis}

Baseline characteristics were collected including age, sex, Ann Arbor stage, primary site location, year of diagnosis, race, ethnicity, use of radiation or chemotherapy, SEER cause-specific death classification, survival months, and vital status. Stage was categorized as early (I-II) or advanced stage (III-IV) for the purpose of this analysis. Primary site location was grouped into the nasal group and the extranasal group. Among the nasal group, cases of the following 
sites were included: upper respiratory tract NOS, nasal cavity, tongue, gum, palate, mouth, nasopharynx, pharynx, oropharynx, submandibular gland, tonsil, Waldeyer's ring, glottis, ethmoid sinus, maxillary sinus, pyriform sinus, and accessory sinus NOS. Among the extra-nasal group, cases of the following sites were included: fundus of stomach, lesser curvature of stomach, duodenum, jejunum, ileum, cecum, small intestine NOS, hepatic flexure of colon, transverse colon, descending colon, overlapping lesion of colon, rectum, GI tract NOS, liver, gallbladder, head of pancreas, main bronchus, upper lobe of lung, lower lobe of lung, overlapping lesion of lung, lung NOS, anterior mediastinum, short bones of the lower limb and associated joints, bones of the skull and face and associated joints, bone marrow, spleen, skin, overlapping lesion of skin, skin NOS, subcutaneous, central portion of the breast, breast NOS, vagina NOS, cervix uteri, descended testis, testis NOS, kidney NOS, lacrimal gland, orbit NOS, cerebrum, frontal lobe, head, and face and neck NOS.

Fisher's exact test was used to compare variables between the two groups. Considering the emergence and application of modern therapies (e.g., asparaginase-based regimens) since 2010, we therefore roughly regarded $1987-2009$ as the old therapy era and 2010-2016 as the modern therapy era. Overall survival (OS) was defined as the time from diagnosis to death from any cause or the last follow-up. Cancer-specific survival (CSS) was defined as the time from diagnosis to death resulting from NKTCL or the last follow-up. Survival curves were constructed using the Kaplan-Meier method. Differences in survival were tested for statistical significance using the log-rank test. Multivariate analysis was conducted using the multivariate Cox model. All statistical analyses were performed using SPSS for Windows (version 18.0; IBM Corporation, Armonk, NY, USA) and GraphPad Prism 7.0 (Graph Pad Software Inc., San Diego, CA, USA). All P values represented were two-sided, and $\mathrm{P}<0.05$ was defined as statistically significant.

\section{Results}

\section{Patient characteristics}

A total of 714 patients with NKTCL were included in this study. The median age of patients at diagnosis was 54 years (IQR, 39-64 years) and $25.82 \%$ of them were older than 60 years. A total of $579(81.55 \%)$ patients showed primary UAT involvement, and 131 (18.45\%) showed primary extra-nasal involvement. Overall, there was a male predominance (462 males and 252 females). Most patients had early-stage disease (Ann Arbor stage I-II, 74.68\%). The majority of the cohort were white $(\mathrm{n}=502,70.80 \%)$. A total of 357 patients were diagnosed from 1987 to 2009 (era 1) and 357 from 2010 to 2016 (era 2). Baseline characteristics of patients from era 1 and era 2 are summarized in Table 1 . The percentage of extra-nasal NKTCLs was lower in era 2 as compared with era $1(13.94 \%$ vs. $22.84 \%, \mathrm{P}=0.0012)$. No significant differences were observed between the two groups in any other clinical parameters.

\section{Survival analysis for NKTCLs}

There were 387 (54.20\%) deaths recorded, with a median follow-up of 15 months. Among them, 326 cases died of NKTCL. The median OS and CSS were 36 and 57 months, respectively (Figure 1A,B). For the entire cohort, there seemed to be a trend towards significance in OS over time $(\mathrm{P}=0.0595)$ (Figure 1C), with a median OS of 24 months for patients diagnosed in era 1 compared with 47 months for those diagnosed in era 2.There was no significant difference in the median CSS for NKTCLs (era 1: 42 months vs. era 2: 72 months, $\mathrm{P}=0.2613$ ) (Figure 1D).

Univariate analysis showed that age $>60$ years old, advanced Ann Arbor stage, primary extra-nasal involvement, and no radiation were significantly associated with shorter OS and CSS (Table 2). Subsequently, these variables were entered into the multivariate models, which further revealed that they maintained their prognostic value for OS (Table 3), while only advanced Ann Arbor stage and no radiation independently predicted worse CSS.

\section{Survival analysis for nasal NKTCLs}

For nasal NKTCLs, the OS of patients diagnosed in era 2 improved as compared with patients diagnosed in era $1(\mathrm{P}=0.0244)$ (Figure $2 A)$, but there was no significant difference in CSS between patients diagnosed in the 2 eras (Figure 2B). We categorized nasal NKTCLs into nasal cavity cases and non-cavity cases according to the primary tumor sites. A total of 376 and 203 cases were classified as nasal cavity cases and non-cavity cases, respectively. Noncavity cases displayed significantly worse OS (median OS: 26 vs. 60 months, $\mathrm{P}=0.0011$ ) and CSS (median CSS: 37 vs. 140 months, $\mathrm{P}=0.0082$ ) compared to nasal cavity cases (Figure 2C,D).

For nasal cavity cases, the OS and CSS were similar 
Table 1 Distribution of the baseline characteristics of 714 patients with extranodal natural killer/T-cell lymphoma by therapeutic eras

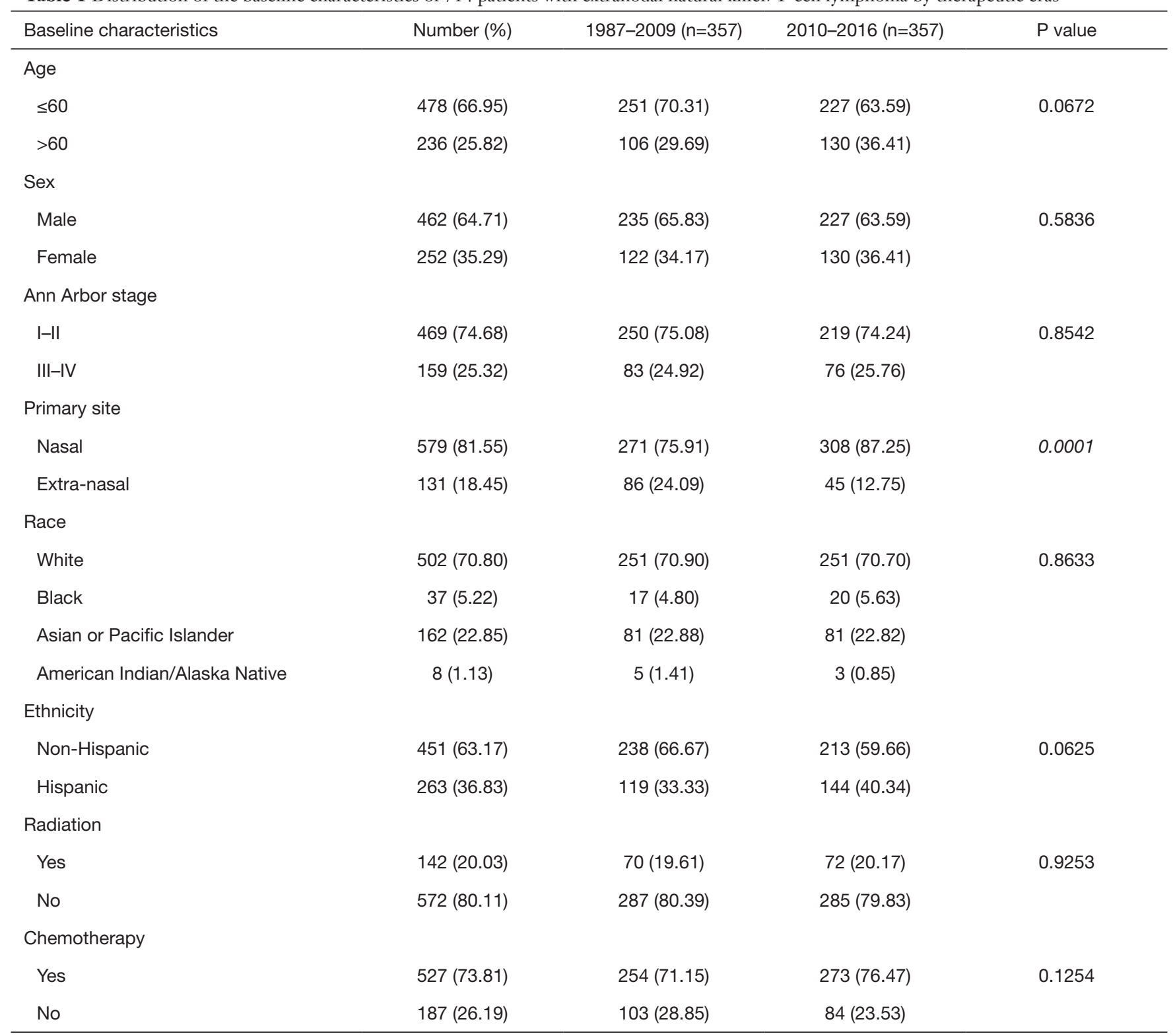

$P$ value $<0.05$ in italic font is statistically significant.

between era 1 and era 2 (Figure $3 A, B$ ). For non-cavity cases, there was a significant improvement in OS from era 1 to era 2 (Figure 3C). The CSS was also improved for patients diagnosed in era 2 as compared with patients diagnosed in era 1 , although the difference was not statistically significant (Figure 3D). For patients with stage I/II nasal disease, there was a significant improvement in OS (median OS: era 155 months vs. era 2 undefined, $\mathrm{P}=0.0018$ ) (Figure $\mathrm{S} 2 \mathrm{~A}$ ). The median CSS was also improved from era 1 to era 2 (median CSS: era 1129.0 months vs. era 2 undefined, $\mathrm{P}=0.0170$ )
(Figure S2B). For patients with stage III/IV nasal NKTCLs, the OS and CSS were similar between the 2 eras (Figure S2C,D). Subsequently, we analyzed the trends in survival for patients with stage I/II non-cavity NKTCLs. We found that patients with stage I/II non-cavity NKTCLs diagnosed in era 2 had a markedly longer OS than those diagnosed in era 1 (median OS: era 115.0 months vs. era 272.0 months, $\mathrm{P}=0.0025$ ) (Figure $3 E$ ). The CSS was also significantly longer for patients diagnosed in era 2 as compared with those diagnosed in era 1 (median CSS: era 137.0 months vs. 

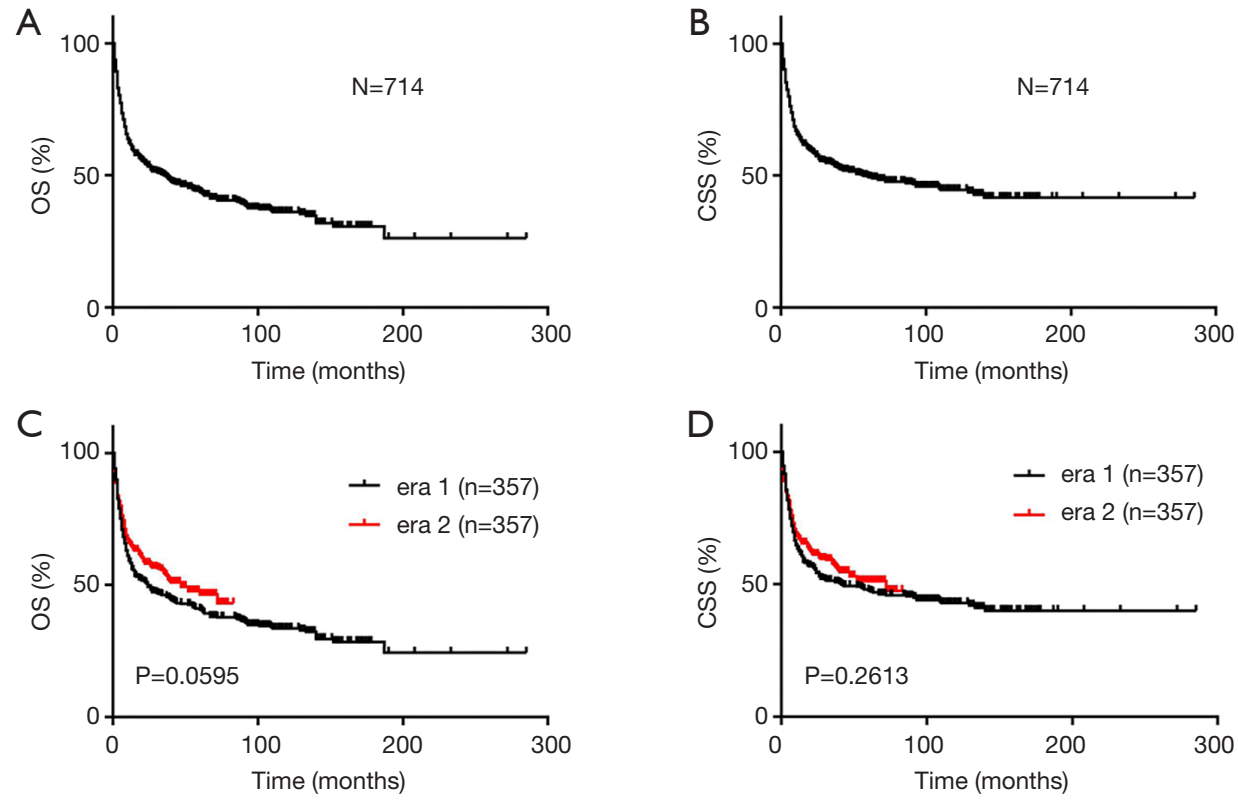

Figure 1 Survival of all the patients with extranodal natural killer/T-cell lymphoma (NKTCL). (A) The overall survival (OS) and (B) cancerspecific survival (CSS) curves for patients with NKTCLs. (C) OS and (D) CSS of patients with NKTCLs by therapeutic eras.

Table 2 Univariate Cox regression analysis of the clinical characteristics for OS and CSS in 714 patients with extranodal natural killer/T-cell lymphoma

\begin{tabular}{|c|c|c|c|c|}
\hline Clinical characteristics & \multicolumn{2}{|c|}{ Univariate analysis (OS) } & \multicolumn{2}{|c|}{ Univariate analysis (CSS) } \\
\hline Sex (female vs. male) & $0.994(0.807-1.223)$ & 0.952 & $1.052(0.840-1.317)$ & 0.658 \\
\hline Year of diagnosis (2010-2016 vs. 1987-2009) & $1.218(0.988-1.502)$ & 0.065 & $1.134(0.907-1.419)$ & 0.270 \\
\hline Age (>60 vs. $\leq 60)$ & $1.462(1.192-1.793)$ & 0.017 & $1.257(1.002-1.575)$ & 0.048 \\
\hline Ann Arbor stage (stage III/IV vs. I/II) & $2.577(2.064-3.218)$ & 0.000 & $2.860(2.258-3.621)$ & 0.000 \\
\hline Radiation (no vs. yes) & $1.571(1.197-2.062)$ & 0.001 & $1.479(1.107-1.977)$ & 0.008 \\
\hline Chemotherapy (no vs. yes) & $1.045(0.836-1.307)$ & 0.700 & $0.837(0.647-1.084)$ & 0.177 \\
\hline Race (White vs. Asian or Pacific Islander vs. others) & $0.956(0.853-1.071)$ & 0.439 & $1.001(0.887-1.130)$ & 0.986 \\
\hline
\end{tabular}

$\mathrm{P}$ value $<0.05$ in italic font is statistically significant. OS, overall survival; CSS, cancer-specific survival; $\mathrm{HR}$, hazard ratio; Cl, confidence interval.

era 2 undefined, $\mathrm{P}=0.0176$ ) (Figure $3 F$ ).

\section{Survival analysis for extra-nasal NKTCLs}

For extra-nasal NKTCLs, no significant improvement from era 1 to era 2 was observed in OS or CSS (Figure $4 A, B$ ).
The OS for extra-nasal NKTCLs was even longer in era 1 than in era 2, however, the difference was not statistically significant. Among the 131 cases of extra-nasal NKTCLs, the most common primary sites were skin $(n=44)$ and the GI tract $(n=30)$. For patients with skin NKTCLs, those diagnosed in era 2 had inferior OS and CSS when compared 
Table 3 Multivariate Cox regression analysis of the clinical characteristics for OS and CSS in 714 patients with extranodal natural killer/T-cell lymphoma

\begin{tabular}{|c|c|c|c|c|}
\hline \multirow{2}{*}{ Clinical characteristics } & \multicolumn{2}{|c|}{ Multivariate analysis (OS) } & \multicolumn{2}{|c|}{ Multivariate analysis (CSS) } \\
\hline & $\mathrm{HR}(95 \% \mathrm{Cl})$ & $P$ value & $\mathrm{HR}(95 \% \mathrm{Cl})$ & $P$ value \\
\hline Age (>60 vs. $\leq 60)$ & $1.307(1.053-1.622)$ & 0.015 & $1.131(0.890-1.437)$ & 0.314 \\
\hline Primary site (extra-nasal vs. nasal) & $1.309(1.012-1.692)$ & 0.040 & $1.242(0.936-1.646)$ & 0.133 \\
\hline Ann Arbor stage (stage III/IV vs. I/II) & $2.366(1.880-2.978)$ & 0.000 & $2.671(2.092-3.411)$ & 0.000 \\
\hline
\end{tabular}

$\mathrm{P}$ value $<0.05$ in italic font is statistically significant. OS, overall survival; CSS, cancer-specific survival; $\mathrm{HR}$, hazard ratio; $\mathrm{Cl}$, confidence interval.
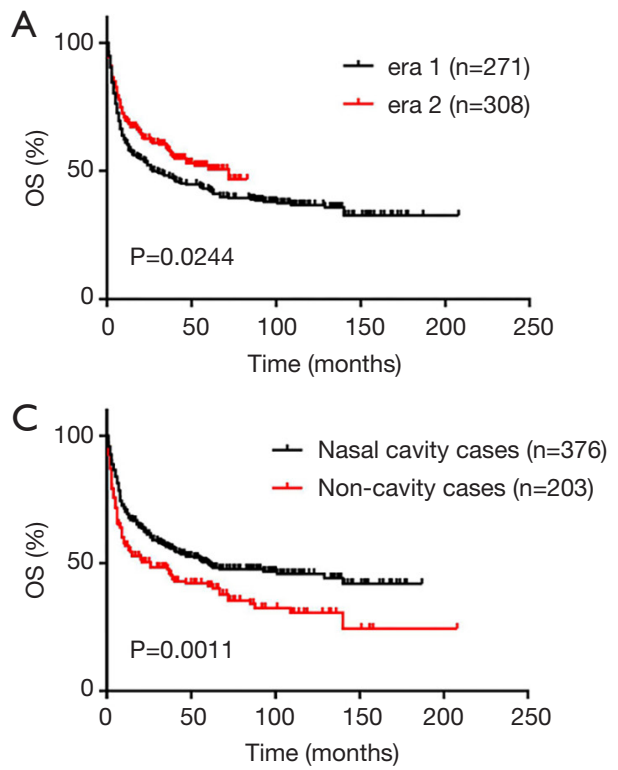
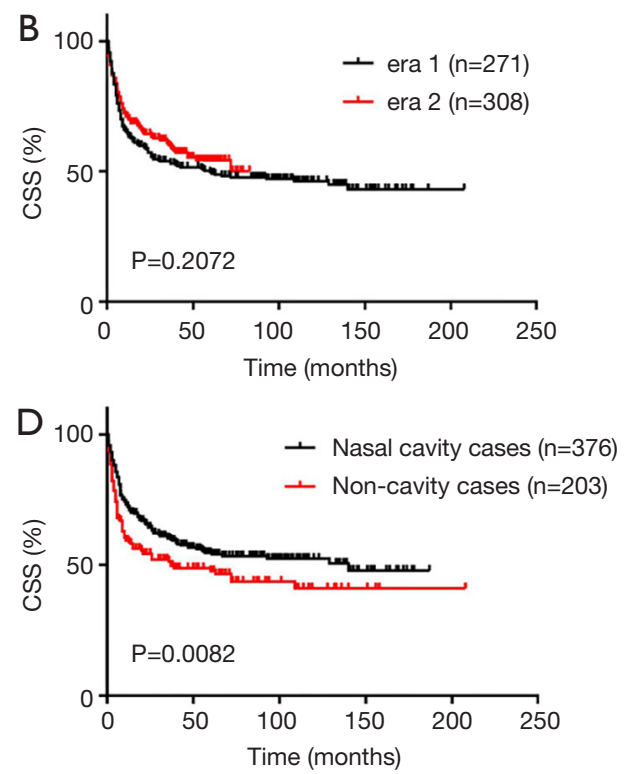

Figure 2 Survival curves for patients with nasal extranodal natural killer/T-cell lymphoma (NKTCL). (A) The overall survival (OS) and (B) cancer-specific survival (CSS) of patients with nasal NKTCLs by therapeutic eras. (C) OS and (D) CSS of patients with nasal cavity NKTCLs and non-cavity nasal NKTCLs.

with those diagnosed in era 1 (Figure $4 C, D$ ). This poorer prognosis of skin NKTCLs in era 2 could be attributed to a higher frequency of stage $\mathrm{V}$ disease in era 2, however, the low number of cases in era 2 prevented us from drawing a conclusion. For patients with GI tract NKTCLs, those diagnosed in era 2 showed similar OS and CSS to those diagnosed in era 1 (Figure 4E,F).

\section{Discussion}

NKTCL is a rare and distinct subtype of non-Hodgkin lymphomas (NHLs) (11). Consistent with previous studies, extra-nasal NKTCLs had significantly worse outcomes than nasal NKTCLs $(8,12,13)$. Although OS for nasal NKTCLs was improved in era 2 compared with era 1 $(\mathrm{P}=0.0244)$, there was no improvement in survival for extranasal NKTCLs, suggesting that cases of nasal origin rather than extra-nasal origin would benefit from the advent of new treatments for NKTCL, which is in accordance with findings from Yamaguchi et al. (8). Moreover, among cases of nasal NKTCL, there was improvement in OS from era 1 to era 2 in non-cavity cases but not in cavity cases, indicating that non-cavity cases are more likely to benefit from modern therapies. 

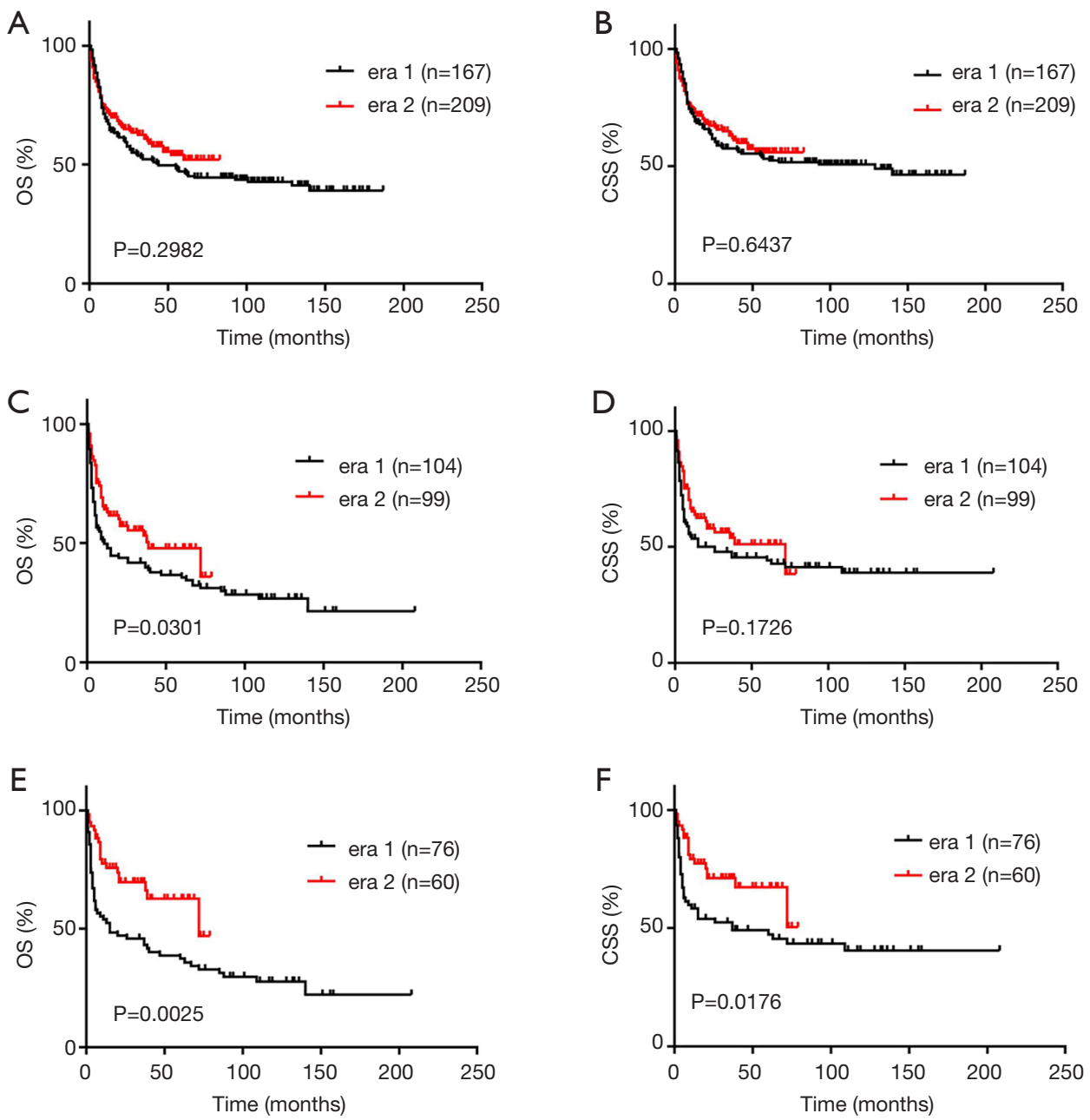

Figure 3 Survival curves for nasal extranodal natural killer/T-cell lymphoma (NKTCL) subgroups by therapeutic eras. (A) The overall survival (OS) and (B) cancer-specific survival (CSS) of patients with nasal cavity NKTCLs. (C) OS and (D) CSS of patients with non-cavity nasal NKTCLs. (E) OS and (F) CSS of stage I/II non-cavity nasal NKTCLs.

In regard to Ann Arbor stage, patients with stage I/II disease have favorable outcomes, while those with stage III/IV disease have markedly worse outcomes, and require additional efforts to obtain durable remission (3). Previous studies have shown that modern asparaginase-based or pegasparaginase-based regimens are associated with superior efficacy compared to conventional anthracycline-based regimens for the treatment of stage I/II disease $(14,15)$. In the present study, nasal NKTCLs in the advanced stage showed inferior survival in both eras. Intriguingly, the OS was significantly improved from era 1 to era 2 in stage I/ II nasal NKTCLs but not stage III/IV nasal NKTCLs, again consistent with the study by Yamaguchi et al. (8). Subsequently, when we restricted our analysis to stage I/II non-cavity NKTCLs, we noted remarkable improvements in OS and CSS in the new era, suggesting that stage I/ II non-cavity nasal NKTCLs constitute a subgroup that benefit most from the use of the modern therapies.

The differences in survival trends among the different subgroups highlight the heterogeneity of NKTCLs. It has long been recognized that extra-nasal NKTCLs have a worse prognosis when compared with nasal NKTCLs. The poor prognosis of extra-nasal NKTCL was partly attributed to the higher frequency of stage III/IV disease in these cases, however, the possible distinct biological characteristics of extra-nasal NKTCLs may also play a role in affecting the prognosis of these cases. According to the study by Yamaguchi et al., only $21 \%$ of patients were fit for the 

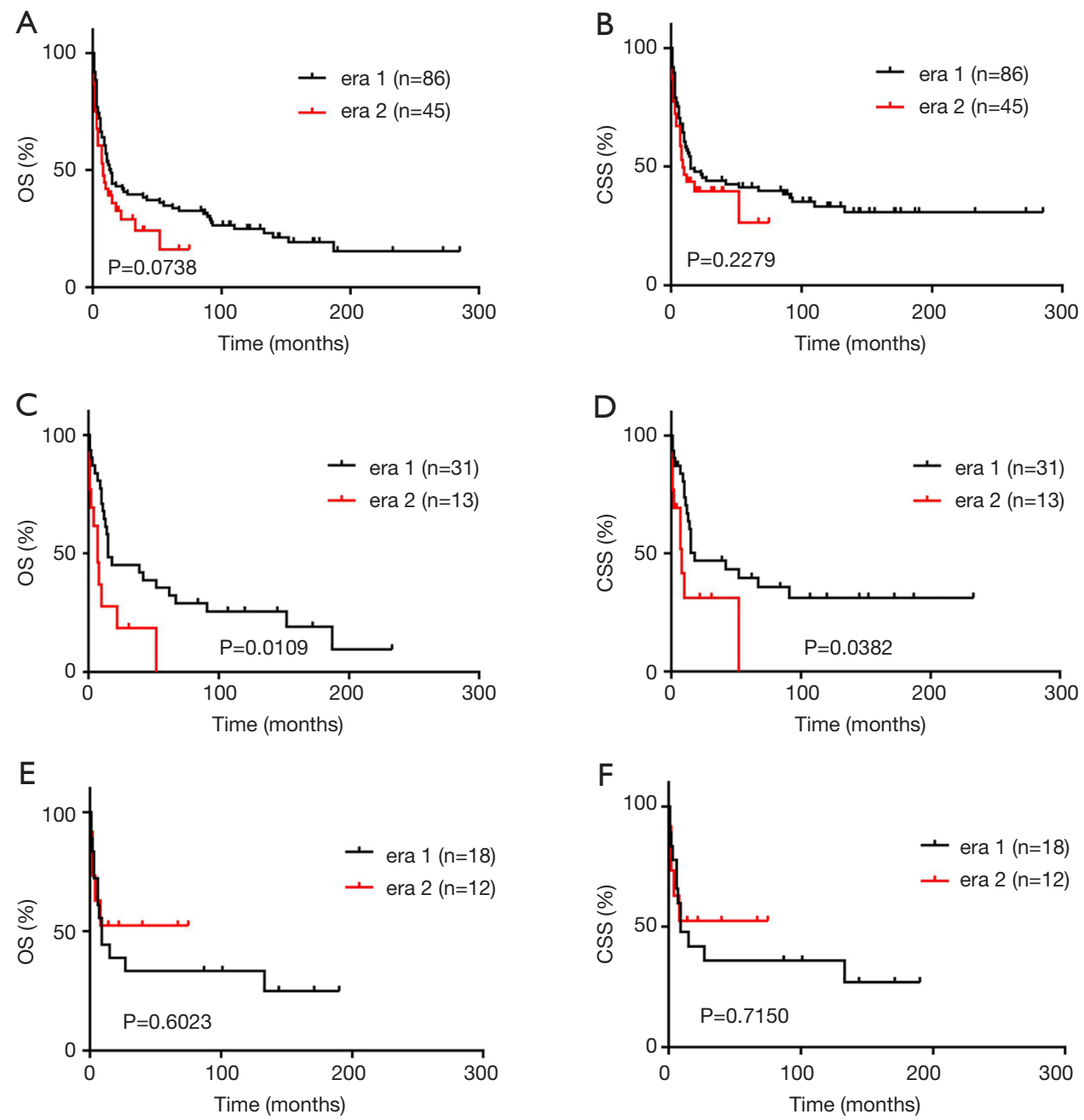

Figure 4 Survival curves for patients with extra-nasal extranodal natural killer/T-cell lymphoma (NKTCL) by therapeutic eras. (A) The overall survival (OS) and (B) cancer-specific survival (CSS) of patients with extra-nasal NKTCLs. (C) OS and (D) CSS of skin NKTCLs. (E) OS and (F) CSS of patients with GI tract NKTCLs.

intensive SMILE regimen, which indicates that only a small proportion of patients with extra-nasal NKTCL benefit from it despite the high efficacy of the SMILE regimen (8). Furthermore, it is possible that even in the patients treated with the SMILE regimen, extra-nasal NKTCLs have a less favorable response when compared with nasal NKTCLs. The disparity in survival trends between cavity and noncavity nasal NKTCLs is also interesting. Although cavity and non-cavity nasal NKTCLs are currently recognized as the same group of diseases, they have distinct clinical and biological characteristics and therefore respond differently to therapies. For example, non-cavity nasal NKTCLs show lower frequencies of granzyme B expression and stage I disease, and a higher frequency of TIA1 expression (9). The improved survival of non-cavity nasal NKTCLs was attributed to the improved outcomes of patients with stage I/II non-cavity nasal disease in the new era. Patients with stage I/II non-cavity nasal NKTCLs may be more likely to benefit from the use of novel radiation modalities including intensity-modulated radiation therapy (IMRT) and the use of chemotherapy.

Intriguingly, the case numbers in era 1 equaled those in era 2 according to the current selection criteria, despite a relatively shorter period of era 2 . We are more inclined to attribute this to an improved diagnostic rate due to better identification of NKTCL. In addition, the nasal- 
to-extra-nasal ratio (81.55\%:18.45\%) was much higher than the reported rate found in an international T-cell lymphoma project study $(68 \%: 26 \% ; 1990-2002)$ (12). This could be due to the increasing and widespread use of more sensitive imaging examinations including PET scans and MRI. Consistently, the frequency of extra-nasal NKTCLs was higher in era 2 than in era 1 . Another possible explanation could be that patients in the SEER cohort are predominantly white. At present, there is limited data on whether clinicopathological features of NKTCLs vary by races or ethnicities. Large-scale study in various ethnicities is warranted to confirm whether our results could fully apply to other ethnic groups or not.

Our study has both clinical and biological implications. As the outcomes of patients with extra-nasal NKTCL have not improved in recent years, both the efficacy and tolerability of front-line treatments for extra-nasal NKTCLs need to be optimized. PD-1 and PD-L1 antibodies show promising therapeutic efficacy and are well tolerated in patients with relapsed/refractory NKTCL $(16,17)$. Combinations of PD-1/PD-L1 inhibitors and less intensive asparaginase-based chemotherapy can be used to treat extra-nasal NKTCLs in the frontline setting. A prospective phase 2 trial (NCT04096690) is currently investigating a PD-1 inhibitor with peg-asparaginase as first-line treatment for advanced stage NKTCL. The prognosis of nasal cavity NKTCLs also needs to be improved, although this group of patients usually has relatively favorable outcomes. For stage I/II nasal cavity NKTCLs, combining PD-1 inhibitors and radiotherapy or chemoradiotherapy can be used to improve the outcomes of patients. A phase 3 randomized trial (NCT04365036) is currently evaluating the addition of a PD-1 inhibitor to chemoradiotherapy in newly-diagnosed earlystage NKTCLs. The differences in prognosis among NKTCLs from different sites suggest that NKTCLs from different anatomical sites may have distinct biological characteristics. Although several studies have explored the genomic landscape of NKTCLs (18-21), the differences in genetic aberrations among NKTCLs from different sites have not been studied. Further studies including more cases are warranted to identify the biological differences among NKTCLs from different sites.

This study is limited by its retrospective nature. Furthermore, some baseline characteristics including the pre-treatment EBV DNA load were not available in the SEER database. The detailed treatment information was also not provided by the SEER database. Therefore, we could not analyze the effects of different treatment regimens on the outcomes of NKTCLs from different sites. Details on deaths not caused by cancer were also unavailable from the SEER database.

\section{Conclusions}

In conclusion, we have demonstrated that the survival of patients with non-cavity nasal NKTCLs, especially those in stage I/II, has improved in the new era, while the survival of patients with nasal cavity NKTCLs and extranasal NKTCLs has not improved. The improvement in the survival of patients with non-cavity nasal NKTCLs could be attributed to the use of chemotherapy and novel radiation modalities. Our study highlights the heterogeneity in clinical outcomes and biology among NKTCLs from different sites. More studies are warranted to define the optimal treatments for patients with NKTCLs.

\section{Acknowledgments}

Funding: This work was supported by the National Natural Science Foundation of China (Grant No. 81720108002), National Major Science and Technology Projects of China (Grant No. 2018ZX09734007), and Translational Research Grant of NCRCH (Grant No. 2020ZKZB01). The funding body played no role in the design of the study and collection, analysis, and interpretation of data and in writing the manuscript.

\section{Footnote}

Reporting Checklist: The authors have completed the STROBE reporting checklist. Available at http://dx.doi. org/10.21037/atm-21-1748

Conflicts of Interest: All authors have completed the ICMJE uniform disclosure form (available at http://dx.doi. org/10.21037/atm-21-1748). The authors have no conflicts of interest to declare.

Ethical Statement: The authors are accountable for all aspects of the work in ensuring that questions related to the accuracy or integrity of any part of the work are appropriately investigated and resolved. All of the SEER data are de-identified and analysis of the data does not require institutional review board approval or informed consent from the patients. The study was conducted in 
accordance with the Declaration of Helsinki (as revised in 2013).

Open Access Statement: This is an Open Access article distributed in accordance with the Creative Commons Attribution-NonCommercial-NoDerivs 4.0 International License (CC BY-NC-ND 4.0), which permits the noncommercial replication and distribution of the article with the strict proviso that no changes or edits are made and the original work is properly cited (including links to both the formal publication through the relevant DOI and the license). See: https://creativecommons.org/licenses/by-nc-nd/4.0/.

\section{References}

1. Chan JK, Sin VC, Wong KF, et al. Nonnasal lymphoma expressing the natural killer cell marker CD56: a clinicopathologic study of 49 cases of an uncommon aggressive neoplasm. Blood 1997;89:4501-13.

2. Nakamura S, Katoh E, Koshikawa T, et al. Clinicopathologic study of nasal T/NK-cell lymphoma among the Japanese. Pathol Int 1997;47:38-53.

3. Tse E, Kwong YL. NK/T-cell lymphomas. Best Pract Res Clin Haematol 2019;32:253-61.

4. Oshimi K, Kawa K, Nakamura S, et al. NK-cell neoplasms in Japan. Hematology 2005;10:237-45.

5. Yamaguchi $M$, Suzuki R, Oguchi $M$. Advances in the treatment of extranodal NK/T-cell lymphoma, nasal type. Blood 2018;131:2528-40.

6. Liu X, Huang E, Wang Y, et al. Dosimetric comparison of helical tomotherapy, VMAT, fixed-field IMRT and 3D-conformal radiotherapy for stage I-II nasal natural killer T-cell lymphoma. Radiat Oncol 2017;12:76.

7. Wang L, Wang ZH, Chen XQ, et al. Firstline combination of gemcitabine, oxaliplatin, and L-asparaginase (GELOX) followed by involvedfield radiation therapy for patients with stage IE/IIE extranodal natural killer/T-cell lymphoma. Cancer 2013;119:348-55.

8. Yamaguchi M, Suzuki R, Miyazaki K, et al. Improved prognosis of extranodal NK/T cell lymphoma, nasal type of nasal origin but not extra-nasal origin. Ann Hematol 2019;98:1647-55.

9. Liu QF, Wang WH, Wang SL, et al. Immunophenotypic and clinical differences between the nasal and extranasal subtypes of upper aerodigestive tract natural killer/T-cell lymphoma. Int J Radiat Oncol Biol Phys 2014;88:806-13.
10. Liang W, He J, Shen Y, et al. Impact of Examined Lymph Node Count on Precise Staging and Long-Term Survival of Resected Non-Small-Cell Lung Cancer: A Population Study of the US SEER Database and a Chinese Multi-Institutional Registry. J Clin Oncol 2017;35:1162-70.

11. Teras LR, DeSantis CE, Cerhan JR, et al. 2016 US lymphoid malignancy statistics by World Health Organization subtypes. CA Cancer J Clin 2016;66:443-59.

12. Au WY, Weisenburger DD, Intragumtornchai T, et al. Clinical differences between nasal and extra-nasal natural killer/T-cell lymphoma: a study of 136 cases from the International Peripheral T-Cell Lymphoma Project. Blood 2009;113:3931-7.

13. Niu SQ, Yang Y, Li YY, et al. Primary site and regional lymph node involvement are independent prognostic factors for early-stage extranodal nasal-type natural killer/ T cell lymphoma. Chin J Cancer 2016;35:34.

14. Wang L, Wang WD, Xia ZJ, et al. Combination of gemcitabine, L-asparaginase, and oxaliplatin (GELOX) is superior to $\mathrm{EPOCH}$ or $\mathrm{CHOP}$ in the treatment of patients with stage IE/IIE extranodal natural killer/ T cell lymphoma: a retrospective study in a cohort of 227 patients with long-term follow-up. Med Oncol 2014;31:860.

15. Wang H, Wuxiao ZJ, Zhu J, et al. Comparison of gemcitabine, oxaliplatin and L-asparaginase and etoposide, vincristine, doxorubicin, cyclophosphamide and prednisone as first-line chemotherapy in patients with stage IE to IIE extranodal natural killer/T-cell lymphoma: a multicenter retrospective study. Leuk Lymphoma 2015;56:971-7.

16. Kwong YL, Chan TSY, Tan D, et al. PD1 blockade with pembrolizumab is highly effective in relapsed or refractory NK/T-cell lymphoma failing l-asparaginase. Blood 2017;129:2437-42.

17. Kim SJ, Lim JQ, Laurensia Y, et al. Avelumab for the treatment of relapsed or refractory extranodal NK/ T-cell lymphoma: an open-label phase 2 study. Blood 2020;136:2754-63.

18. Koo GC, Tan SY, Tang T, et al. Janus kinase 3-activating mutations identified in natural killer/T-cell lymphoma. Cancer Discov2012;2:591-7.

19. Wen H, Ma H, Cai Q, et al. Recurrent ECSIT mutation encoding V140A triggers hyperinflammation and promotes hemophagocytic syndrome in extranodal NK/T cell lymphoma. Nat Med 2018;24:154-64. 
20. Jiang L, Gu ZH, Yan ZX, et al. Exome sequencing identifies somatic mutations of DDX3X in natural killer/ T-cell lymphoma. Nat Genet 2015;47:1061-6.

21. Li Z, Zhang X, Xue W, et al. Recurrent GNAQ mutation

Cite this article as: He L, Zou Y, Tang X, Wang J, Xing L, Zhang J, Li J, Guo J, Miao Y. Survival trends for extranodal NK/T-cell lymphoma, nasal type from different anatomical sites: a population-based study. Ann Transl Med 2021;9(10):849. doi: $10.21037 /$ atm-21-1748 encoding T96S in natural killer/T cell lymphoma. Nat Commun 2019;10:4209.

(English Language Editor: C. Betlazar-Maseh) 


\section{Supplementary}

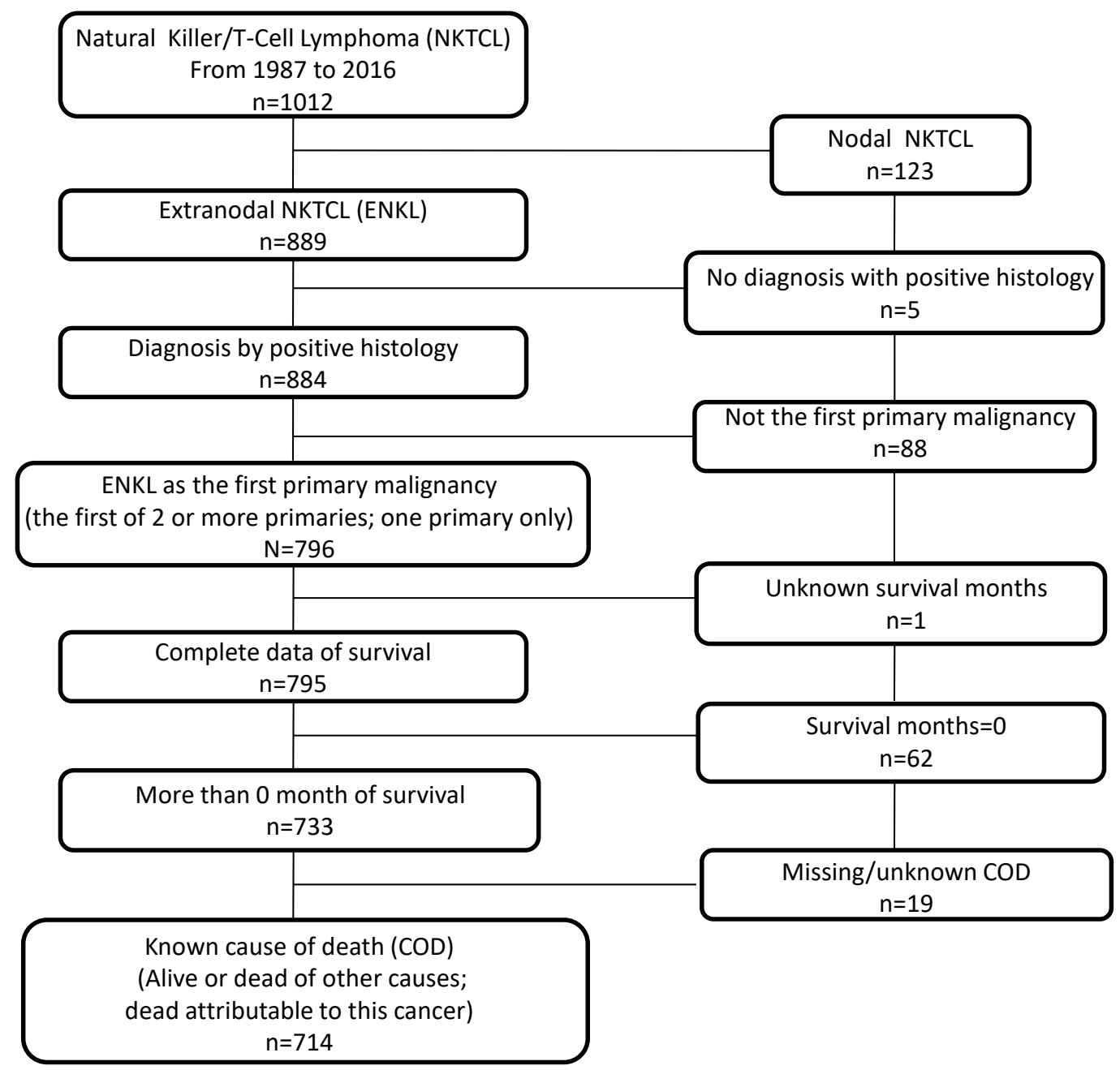

Figure S1 A flowchart of patient selection for the current study. 

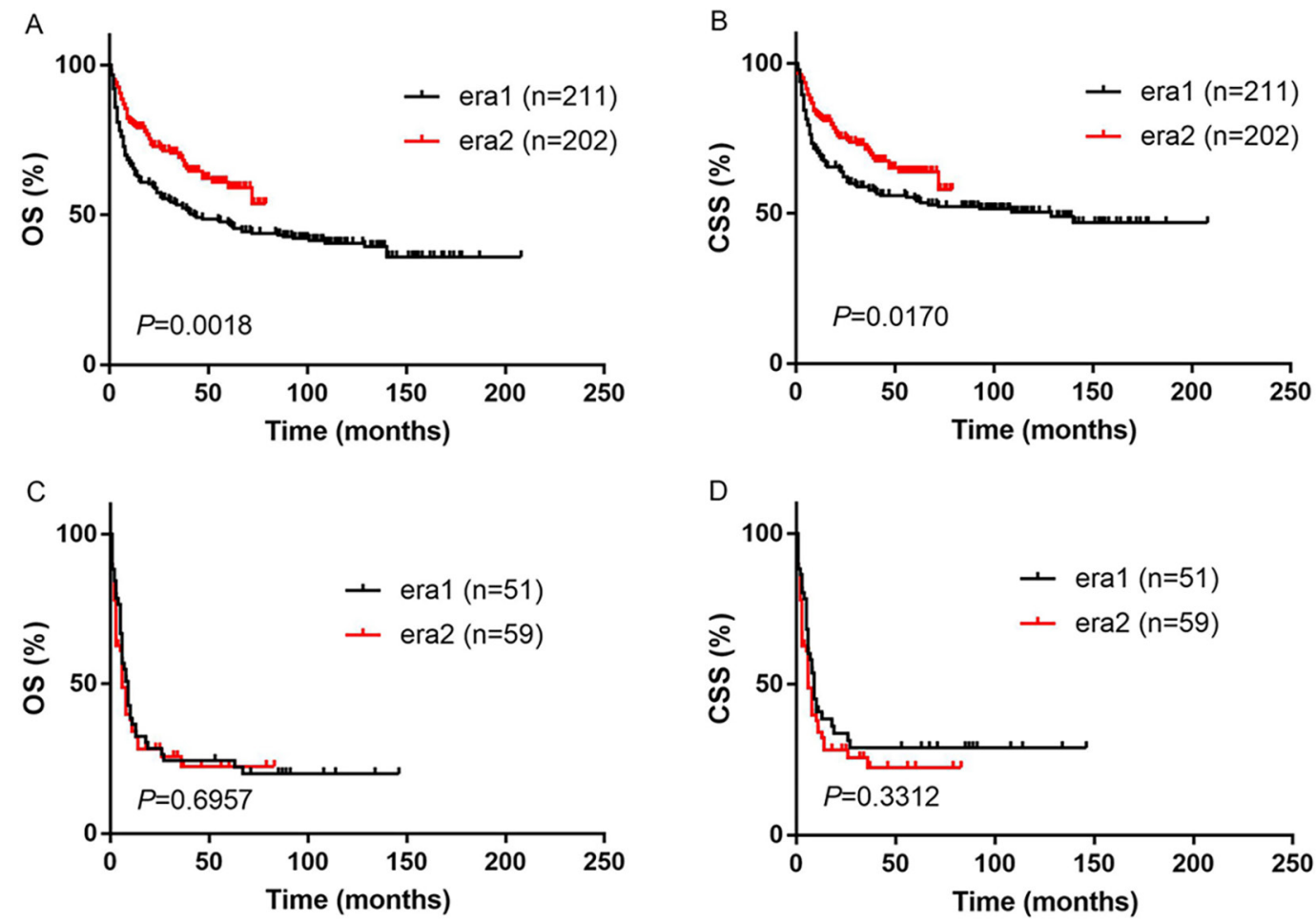

Figure S2 Survival curves for nasal extranodal natural killer/T-cell lymphoma (NKTCL) subgroups by therapeutic eras. (A) The overall survival (OS) and (B) cancer-specific survival (CSS) of patients with stage I/II nasal NKTCLs. (C) OS and (D) CSS of patients with stage III/IV nasal NKTCLs. 